\title{
APPLICATION FOR MEMBERSHIP
}

I am interested in the work of the Society and should like to become a Member.

Name and Address (BLOCK LETTERS)

(Please state Mr., Mrs., Miss, or Title)

I cnclose $£$ : : being my subscription as a Life/ Ordinary Member.*

I enclose a completed Banker's Order.

I enclose a completed Covenant Form.

(Delete any of the above not applicable.)

* The minimum annual subscription is $\oint^{\mathrm{I}}$, but the value to the Society is almost doubled if it is paid under a 7-years Deed of Covenant. The cost to the member of doing this is nothing at all.

Life Membership Fee

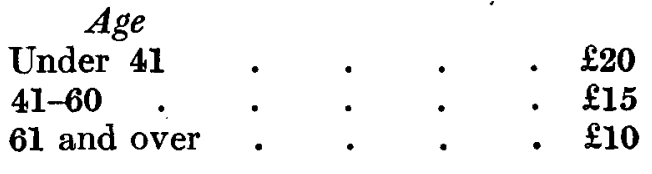




\section{BANKER'S ORDER}

19

(Bank)

(Address)

Please pay for the Credit of THE FAUNA PRESERVATION SOCIETY, Messrs. Barclays Bank, Ltd., Camden Town Branch, my subscription of. due on January Ist, I9 ; and a similar amount on January Ist each year until further notice.

(Signature)

MINIMUM SUBSCRIPTION £I per annum but Members are asked to subscribe as generously as possible. 


\section{THE FAUNA PRESERVATION SOCIETY \\ Patron :}

HER MAJESTY THE QUEEN

President :

The Marquess of Willingdon

\section{Vice-Presidents :}

The Duke of Abercorn, K.G., K.P.

A. Ezra, Esq., O.B.E.

Sir William Gowers, K.C.M.G., C.B.

J. SPEDAN LEWIS, EsQ.

Lt.-Col. J. Stevenson-Hamilton, J.P., D.L., Ll.D.

Dr. Willard G. Van Name, Ph.D.

Hon. Treasurer :

Lan D. Malcolmson, Esq.

Secretary :

Lt.-Col. C. L. Boyle

Assistant Secretary :

Miss Kathleen Clarke

\section{Council :}

The Marquess of Willingdon (Chairman)

Sir William Gowers, K.C.M.G., C.B. (Vice-Chairman)

W. Ameer Ali, EsQ., C.I.E.

G. C. L. Bertram, Esq., M.A., Рh.D.

Captain Keith Caldwell

G. S. Cansdale, EsQ., B.A., B.Sc., F.L.S.

Colonel R. S. Clarke, T.D., D.L., M.P.

G. Dent, Esq., M.C.

Alfred Ezra, Esq., O.B.E.

R. S. R. FitTER, Esq.

Maxwell KNIGHT, EsQ., O.B.E., F.L.S.
I.D. Malcolmson, EsQ.

T. C. S. MorrISON-ScotT, EsQ., D.S.C., M.Sc.

S. A. Neave, EsQ., C.M.G., O.B.E., M.A., D.Sc., F.R.E.S.

Captain C. R. S. Pitman, C.B.E., D.S.O., M.C.

G. F. Herbert Smith, Esq., C.B.E., D.Sc.

Sir T. Shenton Thomas, G.C.M.G., O.B.E.

\section{Bankers :}

Messns. Barclays Bank, Ltd., Camden Town, N.W. 1

\section{Offices:}

c/o Zoological Society of London, Regent's Park, London, N.W. 8

\section{Telephone Number :}

Primrose 3333

\section{Subscriptions :}

Annual Subscription : $£$. Life membership : Age under 41

\section{Notice :}

This Society accepts no responsibility for opinions expressed in individual Articles in this Journal. 